\title{
XPath rewriting using multiple views
}

\author{
Junhu Wang ${ }^{1}$ and Jeffrey $\mathrm{Xu} \mathrm{Yu}{ }^{2}$ \\ 1 Griffith University, Gold Coast, Australia \\ J.Wang@griffith.edu. au \\ 2 Chinese University of Hong Kong, Hong Kong, China \\ yu@se.cuhk.edu.hk
}

\begin{abstract}
We study the problem of tree pattern query rewriting using multiple views for the class of tree patterns in $P^{\{/ /,[]\}}$. Previous work has considered the rewriting problem using a single view. We consider two different ways of combining multiple views, define rewritings of a tree pattern using these combinations, and study the relationship between them. We show that when rewritings using single views do not exist, we may use such combinations of multiple views to rewrite a query, and even if rewritings using single views do exist, the rewritings using combinations of multiple views may provide more answers than those provided by the union of the rewritings using the individual views. We also study properties of intersections of tree patterns, and present algorithms for finding rewritings using intersections of views.
\end{abstract}

\section{Introduction}

Query rewriting using views has many applications including data integration, query optimization, query caching, and support of physical data independence [5]. Given a query, it studies finding another query using only the views to produce correct answers to the original query. In the literature, two types of query rewritings are studied, namely, equivalent rewritings and contained rewritings. Given a view, $V$, and a query, $Q$, an equivalent rewriting produces all answers to the original query $Q$ using view $V$, whereas a contained rewriting may produce a subset of the answers to $Q$ using $V$. Both types of rewritings have been extensively studied in the relational database context [5].

Recently, XML query rewriting has attracted attention because of the rising importance of XML data $[3,14,7,6,2,9]$. XPath lies in the center of all XML languages, where the major classes of XPath expressions that have been studied are tree patterns $[1,8]$. Among previous work on rewriting XPath queries using views, Xu and Ozsoyoglu [14] studied the complexity of finding equivalent rewritings for four types of tree patterns studied in [8] and presented an approach for finding and minimizing such rewritings. Mandhani and Suciu [7] presented, in addition to cache organization, an efficient but incomplete method for finding equivalent rewritings of tree patterns involving $/, / /,[]$ and $*$ when the patterns are assumed to be minimized and may have value-based predicates. In [6], Lakshmanan et al. studied maximal contained rewritings of tree patterns where 


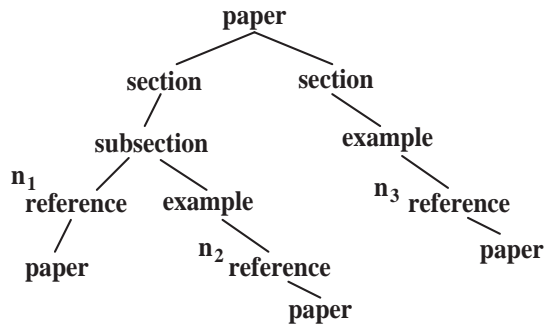

Fig. 1. Example XML tree $t$

both the views and queries are in $P^{\{/ /,[]\}}$, both in the absence and in the presence of non-recursive, non-disjunctive DTDs. As the name implies, the maximal contained rewriting is a rewriting that contains all other contained rewritings.

All of the above works focus on rewriting the query using a single view. In other words, no combination of views is considered for the purpose of rewriting. In this paper we study tree pattern rewriting using multiple views, and our work is motivated by the following observations. Suppose we have a set $\mathcal{V}$ of views and the query $Q$. Then

1. It is possible for $Q$ to have no contained rewritings using any individual view in $\mathcal{V}$, but it can be (partially) answered using the combination of some of the views. As a simple example, the query $Q=\operatorname{article}[$ table] [figure]/author cannot be (partially) answered using either $V_{1}=$ article [table]/author or $V_{2}=$ article [figure]/author, but it can be answered using $V_{1} \cap V_{2}$, which is equivalent to $Q$.

2. Even if $Q$ does have contained rewritings using $V_{1}$, and/or $Q$ does have contained rewritings using $V_{2}$, there may be contained rewritings of $Q$ using some combination of $V_{1}$ and $V_{2}$ (e.g., intersection) which provide strictly more answers to $Q$ than the union of all contained rewritings of $Q$ using the individual views. This is demonstrated by Example 1 .

3. Even if a query $Q$ does not have an equivalent rewriting using $V$ according the conventional definition of rewriting $[6,14,7]$, it is still possible to find all of the correct answers to $Q$ using $V$ for all xML trees. This is demonstrated in Example 2.

The example below demonstrates observation 2 .

Example 1. Consider the views

$V_{1}=$ paper $/ /$ subsection $/ /$ reference,

$V_{2}=$ paper//example/reference

and the query

$Q=$ paper//subsection//example/reference//paper.

It can be verified that

$V_{1} \cap V_{2}=$ paper//subsection//example/reference, 


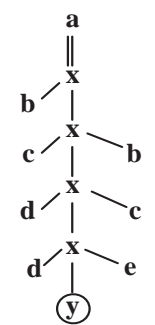

(a) $Q$

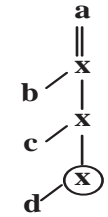

(b) $V$

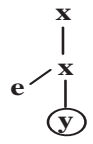

(c) $Q_{1}$

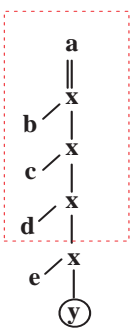

(d) $Q_{1} \circ V$

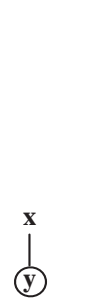

(e) $Q_{2}$

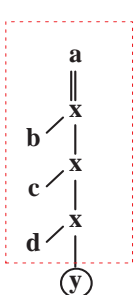

(f) $Q_{2} \circ V$

Fig. 2. $Q$ has no equivalent rewriting using $V$ according to conventional definition, but $Q$ can be fully answered using $V: Q_{1} \circ V \cap Q_{2} \circ V=Q$

and evaluating the pattern reference//paper over the answers of $V_{1} \cap V_{2}$ will produce the same answers as $Q$, that is, reference//paper is an equivalent rewriting of $Q$ using $V_{1} \cap V_{2}$. However, the maximal contained rewriting of $Q$ using $V_{1}$ is reference//example/reference//paper, and the maximal contained rewriting of $Q$ using $V_{2}$ is reference//subsection//example/reference//paper.

As we show next, the rewriting using $V_{1} \cap V_{2}$ produces strictly more answers than the union of the answers produced by the maximal contained rewritings using $V_{1}$ and $V_{2}$ individually. For the XmL tree $t$ shown in Fig.1, evaluating $V_{1}$ over $t$ produces (the subtrees rooted at) $n_{1}$ and $n_{2}$, and evaluating $V_{2}$ over $t$ produces (the subtrees rooted at) $n_{2}$ and $n_{3}$. Therefore, the maximal contained rewriting using $V_{1}$ or $V_{2}$ will produce no answers for $Q$, but the rewriting using $V_{1} \cap V_{2}$ will produce the paper node under $n_{2}$.

The next example demonstrates observation 3 .

Example 2. Consider the query $Q$ and view $V$ shown in Fig.2 (a) and (b) respectively. It can be verified that $Q$ has no equivalent rewritings using $V$ according to the conventional definition. But given any XML tree $t$, we can find $Q(t)$ using the view as follows. We evaluate the query $Q_{1}=x / x[e] / y$ over the subtrees in $V(t)$, and denote the results as $Q_{1}(V(t))$; we then evaluate $Q_{2}=x / y$ over the subtrees in $V(t)$, and obtaining a set denoted as $Q_{2}(V(t))$. Finally, we take the intersection of $Q_{1}(V(t))$ and $Q_{2}(V(t))$. It can be verified that $Q(t)=Q_{1}(V(t)) \cap Q_{2}(V(t))$.

We will study contained and equivalent rewritings of tree patterns in $P^{\{/ /,[]\}}$ using multiple views in the absence and presence of DTDs, with the intersection and union operations. Our main contributions are summarized below.

- We define (contained and equivalent) rewritings of a tree pattern using two different combinations of views, and show the relationship between these rewritings. 
- We show the intersection of some tree patterns, if not empty, can be expressed as the union of tree patterns, and provide an efficient algorithm to translate the intersection into union.

- Based on the above, we provide algorithms for finding the maximal contained rewritings and equivalent rewritings using the intersection of views. We also show the effect of an non-recursive DTD on the rewritings.

The rest of the paper is organized as follows. Section 2 provides the background and notations. Section 3 presents the algorithm for reformulating intersections of tree patterns into union, and studies rewritings using the intersection of views. Section 4 defines rewritings using a different combination views, and compare them with rewritings using the intersection. Section 5 considers the effects of non-recursive DTDs. Section 6 surveys related work. Finally Section 7 concludes the paper.

\section{Preliminaries}

\subsection{XML trees and tree patterns}

Let $\Sigma$ be an infinite set of tags. An XML tree is a tree such that every node is labeled with a tag in $\Sigma$. A tree pattern (TP) is a tree with a unique distinguished node, and with every node labeled with a tag in $\Sigma$, and every edge labeled with either / or //. Such a TP corresponds to an XPath expression involving the child axis, descendant axis, and branching condition []. A tree pattern has a tree representation. Fig.2 show several TPs, and the TP in Fig.2 (b) represents the XPath expression $a / / x[b] / x[c] / x[d]$. Here, single and double lines represent /edges and //-edges respectively. The distinguished node is indicated by a circle. Note: the TPs in our discussion correspond to the fragment $P^{\{/ /,[]\}}$defined in [8]. A subset of $P^{\{/ /,[]\}}$, denoted $P^{\{/ /\}}$, contains all TPs that has a single root-to-leaf path.

Let $\mathcal{T}$ be an XML tree or a TP, and $v$ be a node in $\mathcal{T}$. We will use $N(\mathcal{T})$ to denote the set of all nodes in $\mathcal{T}, r t(\mathcal{T})$ to denote the root of $\mathcal{T}$, and label $(v)$ to denote the label of $v$. For any TP $P, \mathrm{DN}_{P}$ and $\mathrm{DP}_{P}$ denote, respectively, the distinguished node and distinguished path of $P$ (i.e., the path from $r t(P)$ to $\operatorname{DN}_{P}$ ).

A matching of a TP, $P$, in an XML tree, $t$, is a mapping $\delta$ from $N(P)$ to $N(t)$ satisfying the following conditions: (1) root-preserving, i.e., $\delta(r t(P))=r t(t)$, (2) label-preserving, i.e., $\forall v \in N(P)$, label $(v)=\operatorname{label}(\delta(v))$, and (3) structurepreserving, i.e., for every edge $(x, y)$ in $P$, if it is a /-edge, then $\delta(y)$ is a child of $\delta(x)$; if it is a //-edge, then $\delta(y)$ is a descendant of $\delta(x)$, i.e, there is a path from $\delta(x)$ to $\delta(y)$. Each matching $\delta$ produces a subtree of $t$ rooted at $\delta\left(\mathrm{DN}_{P}\right)$, denoted $s u b_{\delta\left(\operatorname{DN}_{P}\right)}^{t}$, which is called an answer to the TP. We use $P(t)$ to denote the answer set of $P$ on $t$ :

$$
P(t)=\left\{s u b_{\delta\left(\mathrm{DN}_{P}\right)}^{t} \mid \delta \text { is a matching of } P \text { in } t\right\} .
$$

Let $T$ be a set of XmL trees. We use $P(T)$ to denote the union of answer sets of $Q$ on the trees in $T$. That is, $P(T)=\bigcup_{t \in T} P(t)$. 


\subsection{Intersection and extension of TPs}

Two tree patterns, $P$ and $Q$, are said to be comparable if label $(r t(P))=\operatorname{label}(r t(Q))$ and label $\left(\mathrm{DN}_{P}\right)=\operatorname{label}\left(\mathrm{DN}_{Q}\right)$. Let $P_{1}, \ldots, P_{n}$ be comparable TPs. For any XML tree $t$, the intersection of $P_{1}, \ldots, P_{n}$, denoted $P_{1} \cap \cdots \cap P_{n}$, returns $P_{1}(t) \cap \cdots \cap$ $P_{n}(t)$. The union of $P_{1}, \ldots, P_{n}$, denoted $P_{1} \cup \cdots \cup P_{n}$, returns $P_{1}(t) \cup \cdots \cup P_{n}(t)$.

Let $P$ and $Q$ be TPs such that label $\left(\operatorname{DN}_{P}\right)=\operatorname{label}(r t(Q))$. The extension of $P$ using $Q$, denoted $Q \circ P$, is the TP obtained by merging $\operatorname{DN}_{P}$ with $\operatorname{rt}(Q)$. The distinguished node of $Q \circ P$ is $\operatorname{DN}_{Q}$. Fig.2 (d) shows the extension of $V$ (Fig.2 (b)) using $Q_{1}$ (Fig.2 (c)). It is easy to see that, for any XML tree $t$, $(Q \circ P)(t)=Q(P(t))$, that is, $(Q \circ P)(t)$ is equivalent to the union of answer sets of $Q$ on the subtrees in $P(t)$.

Let $P_{1}, \ldots, P_{n}$ be comparable TPs, and $Q$ be a TP such that $r t(Q)$ and $\mathrm{DN}_{V_{i}}$ have identical labels. We denote by $Q \circ\left(P_{1} \cap \cdots \cap P_{n}\right)$ the extension of $P_{1} \cap \cdots \cap P_{n}$ using $Q$, which returns, for any $t, Q\left(P_{1}(t) \cap \cdots \cap P_{n}(t)\right)$.

\subsection{TP containment}

Let $P$ and $Q$ be TPs. $P$ is said to be contained in $Q$, denoted $P \subseteq Q$, if for every XML tree $t, P(t) \subseteq Q(t)$. It is shown in [1] that $P \subseteq Q$ iff there is a containment mapping from $Q$ to $P$. Recall: A containment mapping (CM) from $Q$ to $P$ is a mapping $\delta$ from $N(Q)$ to $N(P)$ that is label-preserving, root-preserving as discussed in the last section, structure-preserving (which now means for any /-edge (x,y) in $Q,(\delta(x), \delta(y))$ is a /-edge in $P$, and for any //-edge $(x, y)$, there is a path from $\delta(x)$ to $\delta(y)$ in $P$ ) and is output-preserving, which means $\delta\left(\mathrm{DN}_{Q}\right)=\mathrm{DN}_{P}$. A homomorphism from $Q$ to $P$ is similar to a CM, except there is no requirement of output-preserving.

The following lemma is proved in [13].

Lemma 1. For TPs $P_{1}, \ldots, P_{n}, P \in P^{\{/ /,[]\}}, P \subseteq P_{1} \cup \cdots \cup P_{n}$ iff there is $i \in[1, n]$ such that $P \subseteq P_{i}$.

\section{TP rewriting using intersections of views}

A view is an existing TP. We define rewritings using intersections of views as follows.

Definition 1. Let $Q$ be a query, and $V_{1}, \ldots, V_{n}$ be comparable views. If $V_{1} \cap$ $\cdots \cap V_{n}$ is non-empty, and there exists $Q^{\prime}$ such that label $\left(r t\left(Q^{\prime}\right)\right)=\operatorname{label}\left(\operatorname{DN}_{V_{1}}\right)$, and $Q^{\prime} \circ\left(V_{1} \cap \cdots \cap V_{n}\right) \subseteq Q$, then we say $Q^{\prime} \circ\left(V_{1} \cap \cdots \cap V_{n}\right)$ is a contained rewriting (CR) of $Q$ using $V_{1} \cap \cdots \cap V_{n}$. If $Q^{\prime} \circ\left(V_{1} \cap \cdots \cap V_{n}\right)=Q$, we say $Q^{\prime}$ is an equivalent rewriting (ER) using $V$. The maximal contained rewriting (MCR) of $Q$ using $V_{1} \cap \cdots \cap V_{n}$, denoted $\operatorname{MCR}\left(Q, V_{1} \cap \cdots \cap V_{n}\right)$, is the union of all CRs of $Q$ using $V_{1} \cap \cdots \cap V_{n}$.

Note that when $n=1$, the above definition reduces to those in [6] (CR and MCR) and [14] (ER). 


\subsection{Properties of intersections}

In this section we identify conditions under which the intersection of TPs are not empty, and present algorithms to translate the intersection into the union of TPs. We present these results using two TPs. Generalization to more TPs is simple.

In many cases the intersection of two TPs $P$ and $Q$ is an empty query, that is, it always returns the empty set. For example, when $P=a / b / x$ and $Q=a / c / / x$. The question arises: when is $P \cap Q$ a non-empty query? We have the follow answer to this question.

Lemma 2. $P \cap Q$ is non-empty iff there is a path $P^{\prime}$ in $P^{\{/ /\}}$such that $P^{\prime} \subseteq$ $\mathrm{DP}_{P}$, and $P^{\prime} \subseteq \mathrm{DP}_{Q}$.

The lemma is true because, when there is no DTD, every TP is non-empty, and $P \cap Q$ is non-empty iff $\mathrm{DP}_{P} \cap \mathrm{DP}_{Q}$ is non-empty.

Let's call the path $P^{\prime}$ in lemma 2 a common distinguished path of $P$ and $Q$. If $\mathrm{DP}_{P}$ and $\mathrm{DP}_{Q}$ do not have //-edges, then only when $\mathrm{DP}_{P}=\mathrm{DP}_{Q}$ there is a CDP of $P$ and $Q$, which is $\mathrm{DP}_{P}$ itself. However, if $\mathrm{DP}_{P}$ and $\mathrm{DP}_{Q}$ do have $/ /$-edges, then there may be (infinitely) many CDPs of $P$ and $Q$. For example, if $P=a / / x[b] / / y, Q=a / / x[c] / / y$, then $P$ and $Q$ have the CDPs $a / / x / / y$, $a / / x / / x / / y, a / / x / / x / / x / / y$ and so on. But there are only a finite number of CDPs that are of interest to us. These CDPs are annotated to form annotated CDPs.

Definition 2. Let $P$ and $Q$ be comparable TPs in $P^{\{/ /,[]\}}$. An annotated CDP (ACDP) of $P$ and $Q$ is a $\mathrm{CDP} P^{\prime}$ of $P$ and $Q$ such that (1) every node in $P^{\prime}$ is annotated with either 1, or 2, or both, (2) there is a (unique) CM $m_{P}$ from $\mathrm{DP}_{P}$ to $P^{\prime}$ such that every node in $\mathrm{DP}_{P}$ is mapped a node annotated with 1 (or both 1 and 2), and for every node $v$ in $P^{\prime}$ annotated with 1 (or both 1 and 2), there is a unique node $u$ in $\mathrm{DP}_{P}$ such that $m_{P}(u)=v$, (3) there is a (unique) CM $m_{Q}$ from $\mathrm{DP}_{Q}$ to $P^{\prime}$ such that every node in $\mathrm{DP}_{Q}$ is mapped a node annotated with 2 (or both 1 and 2), and for every node $v$ in $P^{\prime}$ annotated with 2 (or both 1 and 2), there is a unique node $u$ in $\mathrm{DP}_{Q}$ such that $m_{Q}(u)=v$.

Intuitively, an ACDP of $P$ and $Q$ is a path in $P^{\{/ /\}}$which contains exactly one "copy" of $\mathrm{DP}_{P}$ and one "copy" of $\mathrm{DP}_{Q}$ (the nodes annotated with 1 form a copy of $\mathrm{DP}_{P}$, and the nodes annotated with 2 form a copy of $\mathrm{DP}_{Q}$ ) and every node appears in at least one of the copies. Fig.3 shows all ACDPs of $P=a / / x[b] / / y$ and $Q=a / / x[c] / / y$.

Next we present an algorithm that finds all ACDPs of $P$ and $Q$. Let $P_{1}=$ $\mathrm{DP}_{P}$ and $P_{2}=\mathrm{DP}_{Q}$. We can find all ACDPs of $P$ and $Q$ by calling the function $\operatorname{Merge}\left(P_{1}, P_{2}\right)$. In the function, a position in $P_{j}$ refers to either a node or a //-edge in $P_{j}$. Each position in $P_{j}$ is given a unique position number, with the position number of $r t\left(P_{j}\right)$ being 0 , and each subsequent position's position number increases by 1 . The function $\operatorname{Merge}\left(P_{i}, P_{j}\right)$ finds the ACDPs of $P_{i}$ and $P_{j}$ by "inserting" the nodes of $P_{i}$ into $P_{j}$. To do so, it needs to find a position, 


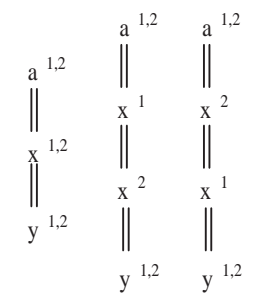

Fig. 3. ACDPs of $a / / x[b] / / y$ and $a / / x[c] / / y$

in $P_{j}$, for every node in $P_{i}$. There are three stages in the process. The first stage (lines 1-6) is to scan $P_{i}$ top-down, marking each node $v$ in $P_{i}$ with a set of markings. Each marking is of the form $\{s: A(v, s)\}$ (except for $\operatorname{rt}\left(P_{i}\right)$, which is marked $\{0\})$, where $s$ is a position, and $A(v, s)$ is a set of positions. The meaning of this marking is that if parent $(v)$ is merged into $P_{j}$ in position $s$, then the possible positions where $v$ can be inserted in $P_{j}$ are those in $A(v, s)$. The criterion to choose the positions in $A(v, s)$ is label-preservation and structurepreservation. The second stage (lines 8-10) removes the impossible positions from the markings, going from bottom-up. The only possible position for $\mathrm{DN}_{P_{i}}$ is the last position in $P_{j}$. Based on structure-preservation, if impossible positions for $v$ are found and deleted, then impossible positions for parent $(v)$ may be found and deleted. In the last stage (lines 13-29), if every node has a possible position, we pick a position $s_{v}$ for each node $v$ in $P_{i}$ according to the markings, and construct a ACDP. For each combination of positions (each combination has one position for every node in $P_{i}$ ) there is a ACDP constructed. Finally the function returns all ACDP constructed this way.

Example 3. Let $P_{1}=a / / x / / y / y$ and $P_{2}=a / / x / / x / / y$. Fig.4 shows the process of running MERGE $\left(P_{1}, P_{2}\right)$.

First, we identify and label each position in $P_{2}$. There are 7 positions labeled $0,1, \ldots, 6$, as shown in the figure. In stage 1 , we mark each node in $P_{2}$ with its possible positions. The $a$-node is marked $\{0\}$. If the $a$-node is put in position 0 , then the possible positions of the next node are $1,2,3,4,5$. Therefore, the first $x$-node is marked with $(0:\{1,2,3,4,5\})$. If the $x$-node is put to position 1 , then the next node may be put to positions 1,3 or 5 , to preserve label and structure of $P_{i}$. If the $x$-node is put to position 2 , then the possible positions for its child are 3 and $5, \ldots$, if the $x$-node is put to position 5 , then its child must be put to position 5 (after the $x$-node). Therefore, the child of the $x$-node is marked with the markings as shown in the figure. Finally, the last node of $P_{1}$ must be put to the last position, position 6 , in $P_{2}$. Thus if its parent is put to position 1 or 3 , then there are no possible positions for it. If its parent is put to position 5 , the it can go to position 6 . This explains the markings of the last node of $P_{1}$. In stage 2 , we remove the impossible positions in the markings. Because position 1 and 3 of the first $y$-node prohibits the second $y$-node to be put in position 6 (as indicated by the markings $(1,\{\})$ and $(3,\{\})$, we know 


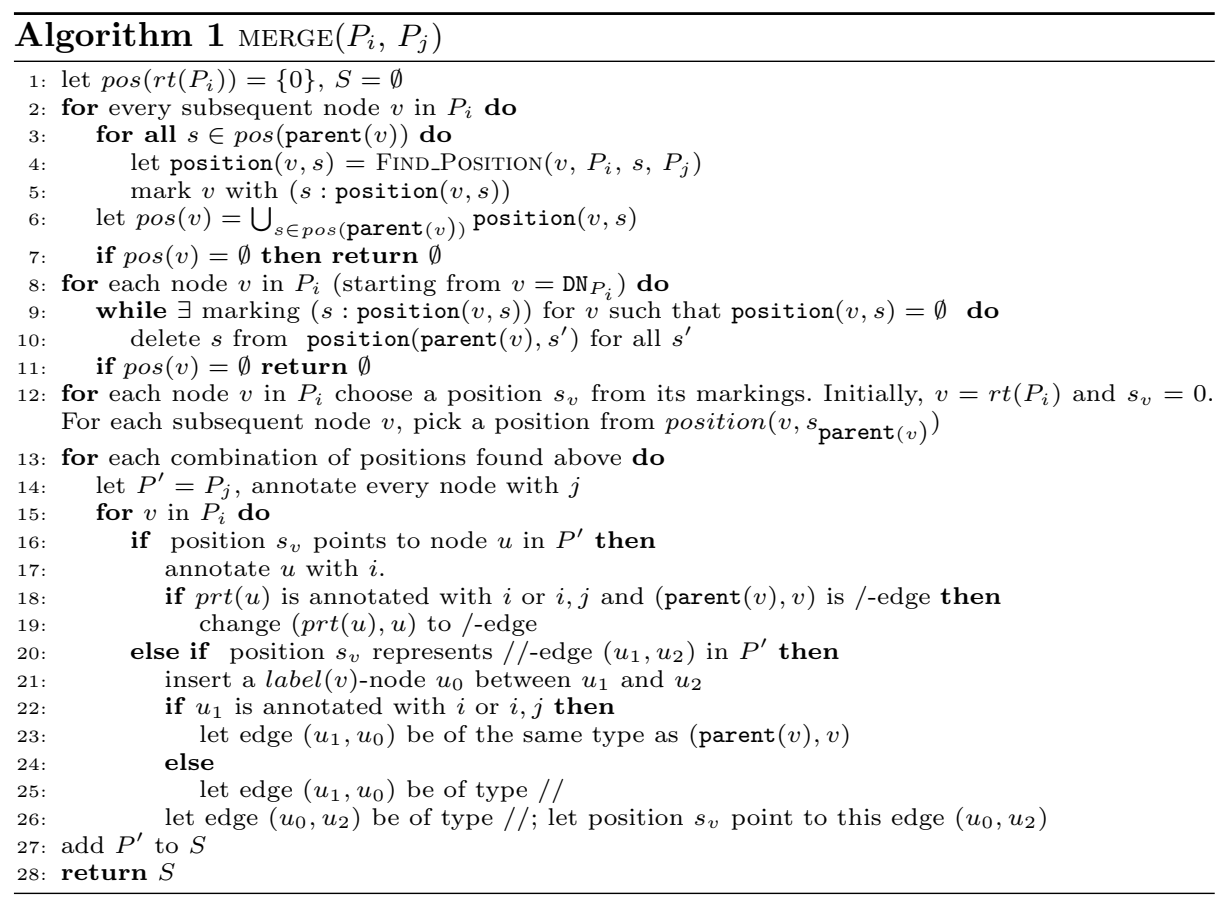

positions 1 and 3 are impossible for the first $y$-node. Thus we delete them from its markings. In stage 3 , for each combination of the positions, we construct an ACDP. A combination of position is made of a position for each node in $P_{1}$. In this example, the combinations of positions are $(0,1,5,6),(0,2,5,6), \ldots,(0,5,5,6)$. We use $(0,5,5,6)$ to explain the construction process. Initially $P^{\prime}=P_{2}$ and every node in $P^{\prime}$ is annotated with 2 . Position 0 points to the root of $P_{2}$, therefore we annotate $r t\left(P_{1}\right)$ with 1 . Position 5 points to a //-edge, therefore we insert an $x$-node, $x_{0}$, in this position and annotate this node with 1 . The edge $\left(x, x_{0}\right)$ and $\left(x_{0}, y\right)$ are to be of type $/ /$ in this case. Now position 5 points to the edge $\left(x_{0}, y\right)$. Since the position for the next node is also 5 , we insert another $x$-node, $x_{1}$, between $x_{0}$ and $y$, and annotate $x_{1}$ with 1 . The last node of $P_{1}$ has position 6 , so we annotate the $y$-node in $P_{2}$ with 1 . Since $x_{1}$ is annotated with 1 , we can change the the edge type of $\left(x_{1}, y\right)$ to that of the corresponding edge in $P_{1}$, in this case, $/$. The resulting $P^{\prime}$ is a ACDP: it is $a^{1,2} / / x^{2} / / x^{2} / / x^{1} / / y^{1} / y^{1,2}$, where the superscripts indicate the annotation. The ACDPs constructed using other combinations are: $a^{1,2} / / x^{1} / / x^{2} / / x^{2} / / y^{1} / y^{1,2}, \quad a^{1,2} / / x^{1,2} / / x^{2} / / y^{1} / y^{1,2}$, $a^{1,2} / / x^{2} / / x^{1} / / x^{2} / / y^{1} / y^{1,2}$, and $a^{1,2} / / x^{2} / / x^{1,2} / / y^{1} / y^{1,2}$.

Number of ACDPs. Let $n$ and $m$ be the number of edges in $P_{i}$ and $P_{j}$ respectively, and $f(m, n)$ be the worst-case number of ACDPs of $P_{i}$ and $P_{j}$ (which occurs when all nodes in $P_{i}$ and $P_{j}$ have the same label, and all edges are //-edges). The following theorem can be proved using induction on $m$ and $n$. 

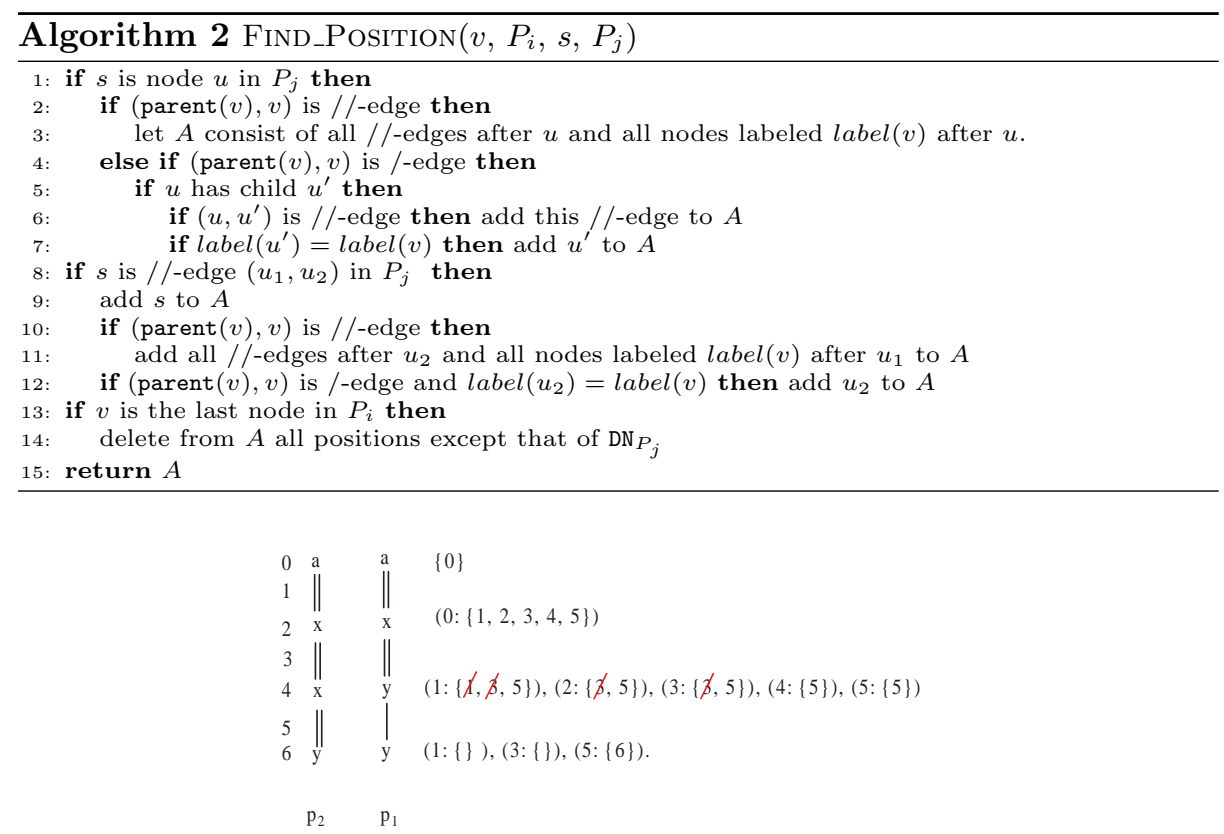

Fig. 4. Finding ADCPs of $P_{1}$ and $P_{2}$

Theorem 1. $f(m, n)=f(n, m)$, which can be calculated recursively as follows:

$$
\begin{aligned}
f(m, n) & =f(m-1, n)+2(f(m-1, n-1) \\
& +f(m-1, n-2)+\cdots+f(m-1,1)) .
\end{aligned}
$$

For example, $f(m, 1)=1, f(2,2)=3, f(3,2)=5, f(3,3)=13, f(4,3)=25$, $f(4,4)=63$ and so on. Thus $f(m, n)$ grows exponentially in general. However, in most practical cases, the number of ACDPs is much smaller than $f(m, n)$.

Complexity. Algorithm $\operatorname{Merge}\left(P_{i}, P_{j}\right)$ runs in $O\left(\left|P_{i}\right| \times\left|P_{j}\right|^{2}\right)$, where $\left|P_{i}\right|$ is the number of nodes in $P_{i}$ : function Find_Position runs in $O\left(\left|P_{j}\right|\right)$, the topdown scan visits each node in $P_{i}$ once, and for each node in $P_{i}$, the function Find_Position is called at most $2\left|P_{j}\right|$ times. The bottom-up scan and the construction of ACDPs can be done in $O\left(\left|P_{i}\right| \times\left|P_{j}\right|\right)$.

Let $P$ be a TP in $P^{\{/ /,[]\}}$. For every node $v$ in $P$, we use $P_{v}$ to denote the subpattern of $P$ rooted at $v$. Let $v$ be a node in $\mathrm{DP}_{P}$, and $u$ be the child of $v$ on $\mathrm{DP}_{P}$ (if $u$ exists). We call the subpattern obtained by removing $P_{u}$ from $P_{v}$ the branching subtree at $v$. For example, in Fig.5 (a), the branching subtrees are indicated by the dotted oval. Next we define merged TPs (MTPs) of $P_{1}$ and $P_{2}$.

Definition 3. Let $P_{1}$ and $P_{2}$ be comparable TPs, and $P^{\prime}$ be an ACDP of $P_{1}$ and $P_{2}$. Let $\delta_{i}$ be the unique CM from $P_{i}$ to $P^{\prime}$ that maps every node in $P_{i}$ to a node in $P^{\prime}$ annotated with $i$. The merged TP $(M T P)$ of $P_{1}$ and $P_{2}$ wrt to $P^{\prime}$ is the 


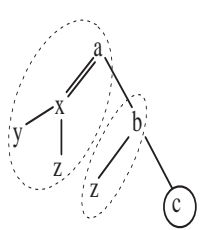

(a) $P_{1}$

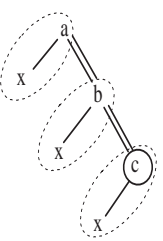

(b) $P_{2}$

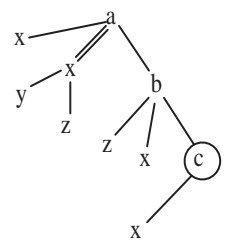

(c) MTP

Fig. 5. TPs $P_{1}, P_{2}$ and the MTP of $P_{1}, P_{2}$

TP obtained as follows: for every node $v$ in $P_{i}$, add the branching subtree at $v_{i}$ under $\delta_{i}\left(v_{i}\right)$.

Fig.5 (c) shows the only MTP of the TPs in Figures 5 (a) and (b).

The following theorem is straightforward.

Theorem 2. Let $P_{1}$ and $P_{2}$ be TPs in $P^{\{/ /,[]\}}$. The union of all MTPs of $P_{1}$ and $P_{2}$ is equivalent to $P_{1} \cap P_{2}$.

Note also that the subpatterns of the MTPs rooted at the distinguished nodes are all identical.

\section{$3.2 \quad$ Finding MCRs using $V_{1} \cap V_{2}$}

Let $Q$ be the query and $V_{1}$ and $V_{2}$ be comparable views. We assume $V_{1} \cap V_{2}$ is not empty, and $V_{1} \nsubseteq V_{2}, V_{2} \nsubseteq V_{1}$. Suppose $V_{1} \cap V_{2}$ is equivalent to $V_{1}^{\prime} \cup \cdots \cup V_{k}^{\prime}$. Clearly $Q^{\prime} \circ\left(V_{1} \cap V_{2}\right) \subseteq Q$ if and only if $Q^{\prime} \circ V_{i}^{\prime} \subseteq Q$ for all $i \in[1, k]$. In other words, $Q^{\prime}$ is a CR of $Q$ using $V_{1} \cap V_{2}$ iff it is a CR of $Q$ using $V_{i}^{\prime}$ for all $i \in[1, k]$. Therefore, to find the MCR of $Q$ using $V_{1} \cap V_{2}$, we can find the MCR of $Q$ using each $V_{i}$ and intersect them. That is,

$$
\operatorname{MCR}\left(Q, V_{1} \cap V_{2}\right)=\bigcap_{i=1}^{k} \operatorname{MCR}\left(Q, V_{i}^{\prime}\right) .
$$

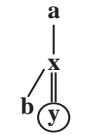

(a) $V_{1}$

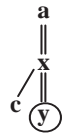

(b) $V_{2}$

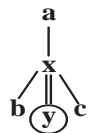

(c) $V_{1}^{\prime}$

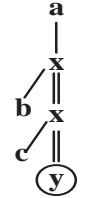

(d) $V_{2}^{\prime}$

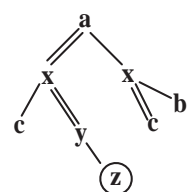

(e) $Q$

Fig. 6. Finding MCR using intersection

Example 4. Consider the views $V_{1}, V_{2}, V_{1}^{\prime}, V_{2}^{\prime}$ and the query $Q$ in Fig.6. $V_{1} \cap V_{2}=$ $V_{1}^{\prime} \cup V_{2}^{\prime}$. We find the MCR of $Q$ using $V_{1}^{\prime}$, which is $y / z$, and the MCR of $Q$ using $V_{2}^{\prime}$, which is also $y / z$. Thus $y / z$ is the MCR of $Q$ using $V_{1} \cap V_{2}$. 


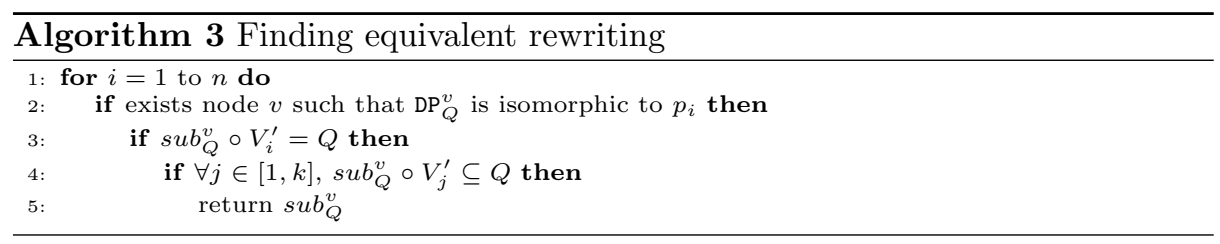

\subsection{Finding ERs using $V_{1} \cap V_{2}$}

Suppose $Q$ has an ER $Q^{\prime}$ using $V_{1} \cap V_{2}$, and $V_{1} \cap V_{2}=V_{1}^{\prime} \cup \cdots \cup V_{k}^{\prime}$, that is, $Q^{\prime} \circ\left(V_{1}^{\prime} \cup \cdots \cup V_{k}^{\prime}\right)=Q$. By Lemma 1 , there exists $i \in[1, k]$, such that $Q^{\prime} \circ V_{i}^{\prime}=Q$. Hence for all $j \in[1, k], Q^{\prime} \circ V_{j}^{\prime} \subseteq Q^{\prime} \circ V_{i}^{\prime}$, and thus there is a CM from $Q^{\prime} \circ V_{i}$ to $Q^{\prime} \circ V_{j}$, so the length of $\mathrm{DP}_{V_{i}^{\prime}}$ cannot be longer than that of $\mathrm{DP}_{V_{j}^{\prime}}$. Furthermore, $\mathrm{DP}_{Q^{\prime} \circ V_{i}^{\prime}}$ is isomorphic to $\mathrm{DP}_{Q}$, and the subpattern of $Q$ rooted at the node that corresponds to $\mathrm{DN}_{V_{i}^{\prime}}$, is an ER of $Q$ using $V_{i}^{\prime}$ (by Lemma 4.8 of [14]).

Using the properties above, we provide a heuristic algorithm, Algorithm 3, for finding ERs using $V_{1} \cap V_{2}$. In the algorithm, we assume $p_{1}, \cdots, p_{n}(n \leq k)$ are the shortest ACDPs of $V_{1}$ and $V_{2}$, and the corresponding MTPs are $V_{1}^{\prime}, \cdots, V_{n}^{\prime 3}$. For any node $v$ on $\mathrm{DP}_{Q}$, the path from $r t(Q)$ to $v$ is denoted $\mathrm{DP}_{Q}^{v}$, and the subpattern rooted at $v$ is denoted $s u b_{Q}^{v}$. The basic idea of the algorithm is as follows. For each shortest ACDP $p_{i}$, we first check whether it is isomorphic to some $\mathrm{DP}_{Q}^{v}$, if not, there is no $\mathrm{ER}$ using $V_{i}^{\prime}$; if yes, we further check whether $s u b_{Q}^{v}$ is an ER using $V_{i}^{\prime}$. If yes, we further check whether the extension of all other MTPs of $V_{1}$ and $V_{2}$ with $s u b_{Q}^{v}$ are contained in $V_{i}$. If yes, $s u b_{Q}^{v}$ is returned as an ER of $Q$ using $V_{1} \cap V_{2}$.

Let $u$ be a node on the distinguished path of $V$. We use $V^{(u)}$ to denote the pattern obtained from $V$ by removing all descendants of $u$ and making $u$ the distinguished node, and $\bar{V}$ to denote $V^{\left(\mathrm{DN}_{V}\right)}$. It is easy to prove the following theorem.

Theorem 3. (1) Suppose $V_{1} \cap V_{2}=V_{1}^{\prime} \cup \cdots \cup V_{n}^{\prime}$. If $Q$ has an ER using $V_{1} \cap V_{2}$, then there is $i \in[1, n]$ such that (i) $V_{i}^{\prime}$ has the shortest distinguished path among $V_{1}^{\prime}, \ldots, V_{n}^{\prime}$, (ii) for all $j \in[1, n]$, there exists $u_{j}$ in $\mathrm{DP}_{V_{j}^{\prime}}$ such that $V_{j}^{\prime\left(u_{j}\right)} \subseteq \overline{V_{i}^{\prime}}$. (2) Algorithm 3 finds the ER if it exists.

Example 5. (1) Consider the views in Fig.6. Since $V_{1} \cap V_{2}=V_{1}^{\prime} \cup V_{2}^{\prime}, \mathrm{DP}_{V_{1}^{\prime}}$ is shorter than $\mathrm{DP}_{V_{2}^{\prime}}$, and there is no node $u$ on the distinguished path of $V_{2}^{\prime}$ such that $V_{2}^{\prime(u)} \subseteq \overline{V_{1}^{\prime}}$, we know there is no ER of $Q$ using $V_{1} \cap V_{2}$ for any $Q$.

(2) Consider the two views $V_{1}$ and $V_{2}$ in Fig.7. There are two MTPs $V_{1}^{\prime}$ and $V_{2}^{\prime}$, and $V_{2}^{\prime} \subseteq V_{1}^{\prime}$. Therefore, $V_{1} \cap V_{2}=V_{1}^{\prime}$. For any TP $Q, Q^{\prime}$ is an ER of $Q$ using $V_{1} \cap V_{2}$ iff $Q^{\prime}$ is an ER using $V_{1}^{\prime}$.

\footnotetext{
${ }^{3}$ Note that although there may be many ACDPs of $V_{1}$ and $V_{2}$, usually there are few shortest ones.
} 


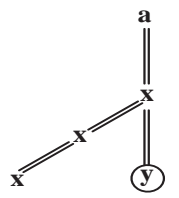

(a) $V_{1}$

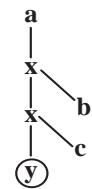

(b) $V_{2}$

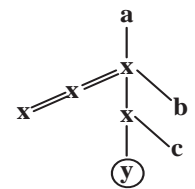

(c) $V_{1}^{\prime}$

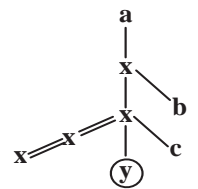

(d) $V_{2}^{\prime}$

Fig. 7. Example for illustrating ER using intersection

\section{Rewriting using other combinations of views}

We now define a second type of rewritings of $Q$ using multiple views that does not require the views to be comparable.

Definition 4. Let $V_{1}, \ldots, V_{n}$ be some views with identical root label (possibly $V_{i}=V_{j}$ for some $\left.i, j\right)$, and $Q$ be a query. If there are $\operatorname{TP} s Q_{1}, \ldots, Q_{n}$ such that $\bigcap_{i=1}^{n}\left(Q_{i} \circ V_{i}\right) \subseteq Q$, and $\bigcap_{i=1}^{n}\left(Q_{i} \circ V_{i}\right)$ is non-empty, then we say $\left\langle Q_{1}, \ldots, Q_{n}\right\rangle$ is a contained rewriting of $Q$ using $\left\langle V_{1}, \ldots, V_{n}\right\rangle$. If $\bigcap_{i=1}^{n}\left(Q_{i} \circ V_{i}\right) \supseteq Q$ also holds, we call the $\mathrm{CR}$ an equivalent rewriting.

Intuitively, if there is a contained rewriting $\bigcap_{i=1}^{n}\left(Q_{i} \circ V_{i}\right)$, then to partially answer $Q$ over $t$, we can evaluate $Q_{i}$ over $V_{i}(t)$ and then find the intersection $\bigcap_{i=1}^{n} Q_{i}\left(V_{i}(t)\right)$. Note that, when $n=1$, the definition reduces to that in [6] (CR and MCR) and [14] .

\subsection{Relationship between rewritings using $\left\langle V_{1}, V_{2}\right\rangle$ and rewritings using $V_{1} \cap V_{2}$}

First, it is easy to prove the following lemma.

Lemma 3. Let $V_{1}$ and $V_{2}$ be comparable views. Then $Q \circ\left(V_{1} \cap V_{2}\right) \subseteq\left(Q \circ V_{1}\right) \cap$ $\left(Q \circ V_{2}\right)$.

However, generally $Q \circ\left(V_{1} \cap V_{2}\right) \nsupseteq\left(Q \circ V_{1}\right) \cap\left(Q \circ V_{2}\right)$. Consider $V_{1}=a / / b / x$ and $V_{2}=a / / c / x$, and $Q=x / / z$. Clearly $V_{1} \cap V_{2}=\emptyset$, hence $Q \circ\left(V_{1} \cap V_{2}\right)=\emptyset$. However, $\left(Q \circ V_{1}\right) \cap\left(Q \circ V_{1}\right) \neq \emptyset$. This example also shows that sometimes even if $V_{1} \cap V_{2}$ is empty, it is still possible to have CRs of $Q$ using $\left\langle V_{1}, V_{2}\right\rangle$, although there are clearly no CRs of $Q$ using $V_{1} \cap V_{2}$.

The next lemma identifies some special cases where $Q \circ\left(V_{1} \cap V_{2}\right)=\left(Q \circ V_{1}\right) \cap$ $\left(Q \circ V_{2}\right)$.

Lemma 4. Let $V_{1}$ and $V_{2}$ be comparable views. If one of the following conditions holds, then $Q \circ\left(V_{1} \cap V_{2}\right)=\left(Q \circ V_{1}\right) \cap\left(Q \circ V_{2}\right)$.

(1) $\mathrm{DP}_{V_{1}}=\mathrm{DP}_{V_{2}}$, and all edges in $\mathrm{DP}_{V_{1}}$ are/-edges.

(2) Every edge in $\mathrm{DP}_{Q}$ is a /-edge.

(3) $V_{1} \subseteq V_{2}$ or $V_{2} \subseteq V_{1}$. 
Using the above lemmas, we can prove the following theorem:

Theorem 4. Let $V_{1}, V_{2}$ be comparable views. For any query $Q$, if there is a $\mathrm{CR}$ of $Q$ using $V_{1} \cap V_{2}$, then there is a $\mathrm{CR}$ of $Q$ using $\left\langle V_{1}, V_{2}\right\rangle$.

Note that the above theorem does not say there is CR using $\left\langle V_{1}, V_{2}\right\rangle$ which contains the CR using $V_{1} \cap V_{2}$. The next example shows that it is possible for $Q$ to have a CR using $V_{1} \cap V_{2}$, and this rewriting is not contained in any CRs of $Q$ using $\left\langle V_{1}, V_{2}\right\rangle$.

Example 6. Let $V_{1}=a / / x / / z, V_{2}=a / / y / z$, and $Q=a / / x / / y / z / / c$. It can be verified that $V_{1} \cap V_{2}=a / / x / / y / z$. Now let $Q^{\prime}=z / / c$, then $Q^{\prime} \circ\left(V_{1} \cap V_{2}\right)=Q$. Thus $Q^{\prime}$ is an ER of $Q$ using $V_{1} \cap V_{2}$.

If there are $Q_{1}$ and $Q_{2}$ such that $\left(Q_{1} \circ V_{1}\right) \cap\left(Q_{2} \circ V_{2}\right) \subseteq Q$, then $\operatorname{DP}_{Q_{1}}$ must be $z / c$ or $z / / c$, and $\mathrm{DP}_{Q_{2}}$ must be $z / c$ or $z / / c$, because $r t\left(Q_{i}\right)$ must be labeled $z$ and $\mathrm{DN}_{Q_{i}}$ must be labeled $c$. One can verify that if $\mathrm{DP}_{Q_{2}}=z / / c$, then $\left(Q_{1} \circ V_{1}\right) \cap\left(Q_{2} \circ V_{2}\right)$ is not contained in $Q$, and if $\mathrm{DP}_{Q_{2}}=z / c$, then $\left(Q_{1} \circ V_{1}\right) \cap\left(Q_{2} \circ V_{2}\right)$ is not equivalent to $Q$ either.

\section{The presence of non-recursive DTDs}

In the following, we assume every TP $P$ is satisfiable under a non-recursive DTD $G$, that is, there is an XML tree $t$ which conforms to $G$, and $P(t) \neq \emptyset$.

In the presence of $G$, no label in an XML tree can appear in a path more than once. Thus any TP that is satisfiable under $G$ cannot have a path that contains two or more nodes with the same label. Therefore, for any comparable views $V_{1}$ and $V_{2}$, there is at most one ACDP of $V_{1}$ and $V_{2}$, and at most one MTP of $V_{1}$ and $V_{2}$. In other words, $V_{1} \cap V_{2}$ is equivalent to a single TP $V^{\prime}$ under $G$. Therefore, to find the MCR or ER of $Q$ using $V_{1} \cap V_{2}$, we only need find the MCR or ER of $Q$ using $V^{\prime}$, and this can be done using the method of [6]. Furthermore, we can prove (see full version of this paper) the following theorem, which implies that, in the presence of $G, Q^{\prime}$ is a CR using $V_{1} \cap V_{2}$ iff $\left\langle Q^{\prime}, Q^{\prime}\right\rangle$ is a CR of $Q$ using $\left\langle V_{1}, V_{2}\right\rangle$.

Theorem 5. Let $V_{1}$ and $V_{2}$ be comparable views, and label $\left(r t\left(Q^{\prime}\right)\right)=$ label $\left(\mathrm{DN}_{V_{1}}\right)$. In the presence of $G, Q^{\prime} \circ\left(V_{1} \cap V_{2}\right)$ is equivalent to $\left(Q^{\prime} \circ V_{1}\right) \cap\left(Q^{\prime} \circ V_{2}\right)$.

\section{More related work}

Besides the recent works discussed in Section 1, several other papers dealt with tree pattern query rewriting. In particular, [10] studied the problem of query answerability using views for general XPath queries, that is, given $Q$ and $V_{1}, \cdots, V_{n}$, whether there are $Q_{1}, \cdots, Q_{n}$ such that $Q_{1} \circ V_{1} \cup \cdots \cup Q_{n} \circ V_{n}=Q$. [3] addressed the problem of answering XPath queries using a single materialized view where, for the view, a combination of node references, typed data values, and full paths may be stored. However, the way in which a query is answered using the view 
is different from ours (and those in Section 1): one can follow node references to go to the original document, so the original XML tree cannot be discarded. [2] studied a different type of equivalent rewriting using multiple views in the presence of structural summaries and integrity constraints: the answer sets of the views are nodes rather than subtrees, and the answers to the new query are obtained by combining answers to the views through a number of algebraic operations. [11] studied correct rewritings of TPs, using a single view, which can be seen as a special form of contained rewritings. [4] attempted to speed-up the finding of MCRs using single views by combining the views into a single tree. [12] studied equivalently answering XPath queries using multiple views based on the assumption that the Dewey codes are stored in the materialized views so that the common ancestors of nodes in different views can be found. Our work is clearly different from all of the above.

\section{Conclusion}

We studied the problem of rewriting TP queries using multiple views for the class $P^{\{/ /,[]\}}$, and defined rewritings using two different combinations of views. We studied the relationship between the two types of rewritings and presented efficient algorithms to reformulate the intersection of TPs into a union of TPs, as well as algorithms for finding the MCRs and ERs using intersections of views. Our definitions and algorithms enable us to make better use of the views in order to answer a query.

Acknowledgement This work is partially supported by Griffith University New Researcher's Grant (GUNRG36621) and grant from the Research Grant Council of the Hong Kong Special Administrative Region, China (CUHK418205). The authors are grateful for helpful comments by Professor Rodney Topor.

\section{References}

1. S. Amer-Yahia, S. Cho, L. V. S. Lakshmanan, and D. Srivastava. Minimization of tree pattern queries. In SIGMOD, 2001.

2. A. Arion, V. Benzaken, I. Manolescu, and Y. Papakonstantinou. Structured materialized views for XML queries. In $V L D B, 2007$.

3. A. Balmin, F. Özcan, K. S. Beyer, R. Cochrane, and H. Pirahesh. A framework for using materialized XPath views in XML query processing. In $V L D B, 2004$.

4. J. Gao, T. Wang, and D. Yang. MQTree based query rewriting over multiple XML views. In $D E X A, 2007$.

5. A. Y. Halevy. Answering queries using views: A survey. $V L D B$ J., 10(4), 2001.

6. L. V. S. Lakshmanan, H. Wang, and Z. J. Zhao. Answering tree pattern queries using views. In $V L D B, 2006$.

7. B. Mandhani and D. Suciu. Query caching and view selection for XML databases. In $V L D B, 2005$.

8. G. Miklau and D. Suciu. Containment and equivalence for an XPath fragment. In PODS, 2002. 
9. N. Onose, A. Deutsch, Y. Papakonstantinou, and E. Curtmola. Rewriting nested XML queries using nested views. In SIGMOD, 2006.

10. K. Tajima and Y. Fukui. Answering XPath queries over networks by sending minimal views. In $V L D B, 2004$.

11. J. Tang and S. Zhou. A theoretic framework for answering XPath queries using views. In XSym, 2005.

12. N. Tang, J. X. Yu, M. T. Özsu, B. Choi, and K.-F. Wong. Multiple materialized view selection for xpath query rewriting. In ICDE, 2008.

13. J. Wang, J. X. Yu, and C. Liu. On tree pattern rewriting using views. In WISE, 2007.

14. W. Xu and Z. M. Özsoyoglu. Rewriting XPath queries using materialized views. In $V L D B, 2005$. 